\title{
GLOBAL ATTRACTORS FOR PARABOLIC P.D.E.'S IN HÖLDER SPACES
}

\author{
By \\ Jan W. Cholewa and Tomasz Dlotko
}

\begin{abstract}
The $2 m$-th order semilinear parabolic p.d.e. is shown to generate a strongly continuous semigroup $T(t)$ of classical solutions on a subspace $X^{\mu}$ of the space of Hölder functions $C^{2 m+\mu}(\mathrm{cl} \Omega)$. Based on the smoothing action of parabolic equations and the dissipativity of $T(t)$ in fractional power spaces the existence of a global attractor for $T(t)$ in the topology of $X^{\mu}$ is then justified. Some applications, e.g. to the famous Cahn-Hilliard equation, are finally discussed.
\end{abstract}

\section{Introduction}

\subsection{Motivation.}

It is well known that the global attractor contains much of the relevant information about dynamics of the system [7]. One of many advantages it offers, resulting from its strong stability properties, is the possibility of controlling the behaviour of the system after a long time. When semiflows generated by p.d.e.'s are considered there are, however, a number of possible choices of phase spaces and consequently properties of the attractor may often be displayed in the frame of various topologies. It might then be important in applications to get an attractor on a possibly 'small' space (i.e. in a strong topology) or, when it is known in a 'larger' space, where its existence is easier to prove, to show that it is the same set in a 'smaller' one. For partial differential equations, this impose the need to study semigroups of very smooth solutions which usually leads to additional technical difficulties. Nevertheless, more regularity of the semigroup

1991 Mathematics Subject Classification. 35K25, 35B40.

Key words and phrases. Parabolic equations, smooth global solutions, dissipative semigroups in Hölder spaces, smooth global attractors.

Supported by the State Committee for Scientific Research Grant No. 2 P301 03205.

Received December 19, 1994.

Revised February 24, 1997. 
still seems to be worth considering, since it provides better control over the asymptotic behaviour of solutions.

Our aim here is just to show the existence of global attractors for semigroups generated on Hölder spaces (i.e. in a possibly strong topology) by initial boundary value problems for autonomous semilinear $2 m$-th order parabolic equations of the form:

(1) $\left\{\begin{array}{l}u_{t}=-\sum_{|\alpha| \leq 2 m} a_{\alpha}(x) D^{\alpha} u+f\left(x, u, D^{1} u, \ldots, D^{k} u\right), \quad t>0, \quad x \in \Omega \subset R^{n} \\ \sum_{|\beta| \leq m_{j}} b_{j \beta}(x) D^{\beta} u=0, \quad m_{j} \leq 2 m-1, \quad j=0, \ldots, m-1, \quad t>0, \quad x \in \partial \Omega \\ u(0, x)=u_{0}(x), \quad x \in \Omega\end{array}\right.$

where $\Omega$ is a bounded domain in $R^{n}$ with $C^{2 m+\mu}$ boundary $\partial \Omega, k \in\{0, \ldots, 2 m-1\}$ and $D^{j}, j=1, \ldots, k$, stays for a vector of partial derivatives with respect to $x$ of order $j$, i.e.

$$
D^{j} u=\left\{D^{\sigma} u\right\}_{|\sigma|=j}
$$

As we have shown in [5], a necessary and sufficient condition for existence of a global attractor for a semigroup generated by (1) on a fractional power space, under some growth restrictions for a nonlinear term $f$, is the asymptotic independence of any 'weak' a priori estimate for solutions of (1) of the initial data. In this paper, basing on the linear theory of parabolic equations [9], [10] and using the abstract approach of [7, Sec. 3.9], [8], dissipativeness of semigroups generated by (1) on smoother phase spaces will be shown under the same assumptions as in [5], except that we naturally need the data of (1) to be slightly more regular and satisfy compatibility conditions necessary for the existence of solutions in the considered classes of functions.

\subsection{Assumptions and basic theoretical tools.}

AsSUMPTION $H_{1}$. Let $f$ be locally Lipschitz continuous with respect to all its arguments, $A=\sum_{|\alpha| \leq 2 m} a_{\alpha}(x) D^{\alpha}$ a uniformly strongly elliptic operator, and additionally let both $A$ and the boundary operators $B_{j}=\sum_{|\beta| \leq m_{j}} b_{j \beta}(x) D^{\beta}$ fulfil regularity assumptions according to [10, p. 1287 (2), (3)]. More precisely, for

(2) $\mu_{0}=\frac{v_{0}^{k+1}}{\left(1+v_{0}\right)^{k}}, \quad$ where $\quad v_{0}=1-\frac{n}{p_{0}} \quad$ and $p_{0}>n$ is fixed from now on, 
(i) $\Omega \subset R^{n}$ is a bounded domain with $C^{2 m+\mu_{0}}$ boundary $\partial \Omega$,

(ii) each $a_{\alpha}(|\alpha| \leq 2 m)$ is an element of $C^{\mu_{0}}(\mathrm{cl} \Omega)$,

(iii) $b_{j \beta}\left(|\beta| \leq m_{j}, j=0, \ldots, m-1\right)$ belongs to $C^{2 m-m_{j}+\mu_{0}}(\partial \Omega)$,

(iv) roots, normality, complementing conditions of $[10, \mathrm{p} .1287]$ hold.

As a consequence of the above requirements $\left(A,\left\{B_{j}\right\}_{j=0}^{m-1}, \Omega\right)$ forms a regular elliptic boundary value problem (cf. [6, Part $1, \S 19]$ ), so that (1) is known to fall into a class of abstract evolutionary equations:

$$
\left\{\begin{array}{l}
\dot{u}+A_{p} u=F_{p}(u), \quad t>0 \\
u(0)=u_{0}
\end{array}\right.
$$

where for each $p \in(1,+\infty)$ an operator

$$
A_{p}=A+k_{0} I
$$

(here $k_{0}>0$ is sufficiently large and independent of $p$ ) considered on the domain

$$
D\left(A_{p}\right)=W_{\left\{B_{j}\right\}}^{2 m, p}(\Omega)=\left\{\phi \in W^{2 m, p}(\Omega): \forall_{j=0, \ldots, m-1} B_{j} \phi=0 \quad \text { on } \quad \partial \Omega\right\}
$$

is sectorial in $L^{p}(\Omega)$. Furthermore (cf. [6, Th. 19.2, 19.3), also [12, $\S 5.5 .1$, §4.9.1]):

$$
A_{p} \text { has compact resolvent, }
$$$$
A_{p} \text { is an isomorphism from } D\left(A_{p}\right) \text { onto } L^{p}(\Omega) \text {, }
$$

and

$$
A_{p} \text { is positive, i.e. } \operatorname{Re}\left(\sigma\left(A_{p}\right)\right)>a \text { for some } a>0 .
$$

According to $[7$, p. 72$]$, [8, Chapt. 3]:

Definition. By a $D\left(A_{p}^{\alpha}\right)$-solution of (4) we understand a continuous function $u:\left[0, \tau_{u_{0}}\right) \rightarrow D\left(A_{p}^{\alpha}\right)$ satisfying $(4)$, such that $(d u / d t):\left(0, \tau_{u_{0}}\right) \rightarrow L^{p}(\Omega)$ is continuous and $u(t)$ belongs to $D\left(A_{p}\right)$ for each $t \in\left(0, \tau_{u_{0}}\right)$.

Assumption $H_{2}$. Let $p_{0} \in(n, \infty)$ and $\alpha_{0} \in(0,1)$ satisfy inequality

$$
k<2 m \alpha_{0}-\frac{n}{p_{0}} \text {. }
$$

Since the above assumption ensures that $D\left(A_{p_{0}}^{\alpha_{0}}\right)$ is embedded in $W^{k, \infty}(\Omega)$, the nonlinear term $F_{p_{0}}: D\left(A_{p_{0}}^{\alpha_{0}}\right) \rightarrow L^{p_{0}}(\Omega)$, (which is an abstract counterpart of a 
locally Lipschitz continuous function $f+k_{0} I$ in (1)) is Lipschitz continuous on bounded subsets of $D\left(A_{p_{0}}^{\alpha}\right)$ and, for each $\alpha \in\left[\alpha_{0}, 1\right)$, (4) is known to have a unique $D\left(A_{p_{0}}^{\alpha}\right)$-solution on a maximal interval of existence $\left[0, \tau_{\max }\left(u_{0}\right)\right)$.

To justify global existence of the $D\left(A_{p_{0}}^{\alpha}\right)$-solution $u$ of (4) we need further:

Assumption $H_{3}$. Let for some $l \geq 0, r \geq 1$

$$
D\left(A_{p_{0}}\right) \subset W^{l, r}(\Omega),
$$

and the following a priori $W^{l, r}(\Omega)$ estimate of solutions be known:

$$
\|u(t)\|_{W^{l, r}(\Omega)} \leq c_{1}\left(u_{0}\right), \quad t>0 .
$$

Let additionally the function $f=f\left(x, u, D^{1} u, \ldots, D^{k} u\right)$ have a power growth

$$
\left|f\left(x, u, D^{1} u, \ldots, D^{k} u\right)\right| \leq c\left(1+\sum_{j=0}^{k}\left|D^{j} u\right|^{\gamma_{j}}\right)
$$

with exponents $\gamma_{j}$ satisfying:

$$
\begin{aligned}
& 1 \leq \gamma_{j}<1+\frac{2 m-j}{j-l+(n / r)} \quad \text { if } \quad r(l-j)<n, \\
& 1 \leq \gamma_{j} \text { arbitrarily large if } n \leq r(l-j) .
\end{aligned}
$$

In the presence of Assumptions $H_{1}, H_{3}$ for $\alpha_{0}$ chosen in (9) there is $\alpha_{1} \in\left[\alpha_{0}, 1\right)$ (see [5, Rem. 1] and references cited there) such that $F_{p_{0}}$ is subordinated to $A_{p_{0}}^{\alpha}$ with any $\alpha \in\left[\alpha_{1}, 1\right)$ according to the condition:

$$
\left\|F_{p_{0}}(u(t))\right\|_{L^{p_{0}}(\Omega)} \leq g\left(\|u(t)\|_{W^{, r}(\Omega)}\right)\left(1+\left\|A_{p_{0}}^{\alpha} u(t)\right\|_{L^{p_{0}}(\Omega)}^{\theta}\right), t>0,
$$

which holds with some $\theta=\theta\left(\alpha_{1}\right) \in[0,1)$ and an increasing function $g: R^{+} \rightarrow R^{+}$. Under Assumptions $H_{1}-H_{3}$ we then know from [5, Th. 1], that for all $\alpha \in\left[\alpha_{1}, 1\right)$ :

Proposition 1. The solution $u$ of the problem (4) corresponding to $u_{0} \in$ $D\left(A_{p_{0}}^{\alpha}\right)$ exists globally for $t \geq 0$ and $T(t) u_{0}=u\left(t, u_{0}\right), t \geq 0$, defines a strongly continuous semigroup of operators $T(t): D\left(A_{p_{0}}^{\alpha}\right) \rightarrow D\left(A_{p_{0}}^{\alpha}\right), t \geq 0$.

Assumption $H_{4}$. Let $c_{1}$ in the estimate (11) be bounded on bounded subsets of $D\left(A_{p_{0}}^{\alpha_{1}}\right)$.

In the presence of Assumption $H_{4}$ and the property (6) mentioned above [5, Th. 1] ensures further that for each $\alpha \in\left[\alpha_{1}, 1\right)$ : 
Proposition 2. The semigroup $T(t): D\left(A_{p_{0}}^{\alpha}\right) \rightarrow D\left(A_{p_{0}}^{\alpha}\right)$ takes bounded sets into bounded sets and is compact for $t>0$. Moreover, if for some subset $V$ of $D\left(A_{p_{0}}^{\alpha}\right)$ a priori estimate (11) is asymptotically independent of $u_{0} \in V$, that is

$$
\exists_{\text {const } .>0} \forall_{u_{0} \in V} \limsup _{t \rightarrow+\infty}\left\|u\left(t, u_{0}\right)\right\|_{W^{l, r}(\Omega)} \leq \text { const. },
$$

then there is a bounded subset $\mathscr{P}$ of $D\left(A_{p_{0}}^{\alpha}\right)$ attracting each point of $V$.

Thus we take finally:

Assumption $H_{5}$. For some $\alpha^{\prime} \in\left[\alpha_{1}, 1\right)$ there is a closed subset $V$ of $D\left(A_{p_{0}}^{\alpha^{\prime}}\right)$ such that a priori estimate (11) is asymptotically independent of $u_{0} \in V$ and $T(t) V \subset V$ for $t \geq 0$.

Under the last assumption Hale's theory of dissipative systems [7, Sec. 4.2] and Propositions 1, 2 guarantee (cf. [5, Th. 2])) that for each $\alpha \in\left[\alpha^{\prime}, 1\right)$, the semigroup $T(t)$ generated by (1) on a complete metric subspace $D\left(A_{p_{0}}^{\alpha}\right) \cap V$ of $D\left(A_{p_{0}}^{\alpha}\right)$ has a global attractor $\mathscr{A}_{\alpha} \subset D\left(A_{p_{0}}^{\alpha}\right) \cap V$.

Let us introduce the space of Hölder functions $C_{\left\{B_{j}\right\}}^{2 m+\mu}(c l \Omega)$;

$$
C_{\left\{B_{j}\right\}}^{2 m+\mu}(c l \Omega)=\left\{\phi \in C^{2 m+\mu}(c l \Omega): \forall_{j=0, \ldots, m-1} B_{j} \phi=0 \quad \text { on } \quad \partial \Omega\right\}
$$

and define for $\mu \in\left(0, \mu_{0}\right)$ a Banach space

$$
X^{\mu}=c l_{C^{2 m+\mu}(c l \Omega)}\left(\bigcup_{\mu<\nu} C_{\left\{B_{j}\right\}}^{2 m+v}(c l \Omega)\right),
$$

where a norm in $X^{\mu}$ is the usual $C^{2 m+\mu}(c l \Omega)$ norm.

DeSCRIPTION OF MAIN RESUlt. We shall show in Theorems 1, 2 that, under Assumptions $\mathrm{H}_{1}-\mathrm{H}_{4}$, (1) generates a $C^{0}$-semigroup of global solutions both on $D\left(A_{p_{0}}^{\alpha}\right)\left(\alpha_{1} \leq \alpha<1\right)$ and on $X^{\mu}\left(0<\mu<\mu_{0}\right)$ phase spaces. If, in addition, Assumption $\mathrm{H}_{5}$ is satisfied, then there exist global attractors $\mathscr{A}_{\alpha}, \mathscr{A}_{\mu}$ corresponding to the semigroup considered on $D\left(A_{p_{0}}^{\alpha}\right) \cap V$ or $X^{\mu} \cap V$ respectively. Furthermore, these attractors coincide, that is $\mathscr{A}_{\alpha}=\mathscr{A}_{\mu}=\mathscr{A}$ for $\alpha \in\left[\alpha^{\prime}, 1\right), \mu \in\left(0, \mu_{0}\right)$.

REMARK 1. The space $C_{\left\{B_{j}\right\}}^{2 m+\mu}(c l \Omega)$ consists of $C^{2 m+\mu}(c l \Omega)$ functions satisfying boundary conditions stated in (1). Hence, if only the set of boundary conditions $\left\{B_{j}\right\}$ does not contain zero order operator then for initial functions from $C_{\left\{B_{j}\right\}}^{2 m+\mu}(c l \Omega)$ compatibility conditions of [10, p. 1288], necessary for existence 
of Hölder $C^{1+(\mu / 2 m), 2 m+\mu}([0, \tau] \times c l \Omega)$ solutions of $(1)$, are satisfied. In the case when $B_{0}$ has the order $m_{0}=0$, additionally to usual compatibility conditions any initial function $u_{0}$ should also satisfy:

$$
B_{0}\left[-A u_{0}+f\left(x, u_{0}, D^{1} u_{0}, \ldots, D^{k} u_{0}\right)\right]=0 \quad \text { on } \quad \partial \Omega .
$$

Therefore, in the discussed case when $B_{0}$ has the order $m_{0}=0$, we shall also require that for $\phi \in C_{\left\{B_{j}\right\}}^{2 m+\mu}(c l \Omega)$

$$
B_{0}[A \phi]=0 \quad \text { on } \quad \partial \Omega
$$

and that the function $f$ satisfies the following implication:

$$
B_{0}\left[f\left(x, \phi, D^{1} \phi, \ldots, D^{k} \phi\right)\right]=0 \quad \text { on } \quad \partial \Omega \quad \text { whenever } \quad B_{0} \phi=0 \text { on } \partial \Omega .
$$

RemarK 2. Since a lot of assumptions have been imposed so far on the problem (1), some comments seem to be necessary. Assumptions $H_{1}, H_{2}$ guarantee local $D\left(A_{p_{0}}^{\alpha}\right)$-solvability of (4). Assumption $H_{3}$ covers possibly the widest class of problems having all solutions globally defined (although, in the special case $m=1$ also weak inequality for $\gamma_{j}$ in the first condition of (13) is admissible). Assumption $H_{4}$ ensures that the semigroup $T(t)$ of $D\left(A_{p_{0}}^{\alpha}\right)$-solutions has orbits of bounded sets bounded, whereas property (6) guarantees that $T(t)$ is compact for $t>0$. Assumption $H_{5}$, in the presence of the results of [5], is necessary for existence of a global attractor for $T(t)$ in fractional power spaces. Therefore, dissipativeness of the semigroups generated by (1) on phase spaces equipped with stronger topologies than those considered in [5] is studied in this paper under stronger than in [5] conditions (i)-(iii) of Assumption $H_{1}$ in which extra $\mu_{0}$-Hölder regularity of the data is expressed.

At the end of this paragraph it should also be mentioned that since (1) is a regular parabolic initial boundary value problem, (i.e. Assumption $H_{1}$ hold), whenever $u_{0}$ and a composite function $f$ in (1) are shown to have appropriate regularity and satisfy required compatibility conditions, the linear theory of $[9$, Chapt. VII, §10, Th. 10.4] and [10, Th. 4.] can be applied to (1) and the following estimates become valid:

$$
\begin{gathered}
\|u\|_{C^{1+(\mu / 2 m), 2 m+\mu}([0, \tau] \times c l \Omega)} \leq c(\tau)\left(\|f\|_{C^{\mu / 2 m, \mu([0, \tau] \times c l \Omega)}}+\left\|u_{0}\right\|_{C^{2 m+\mu}(c l \Omega)}\right) \\
\|u\|_{W_{q}^{1,2 m}((0, \tau) \times \Omega)} \leq \operatorname{const}\left(\|f\|_{L^{q}((0, \tau) \times \Omega)}+\left\|u_{0}\right\|_{B_{q}^{2 m-2 m / q}(\Omega)}\right)
\end{gathered}
$$


where $c$ in (21) is a continuous and increasing function of $\tau$. Moreover, according to [10, Th. 5.2], for each $\mu \in\left(0, \mu_{0}\right)$ the set

$$
\mathscr{D}=\left\{\phi \in \bigcap_{p>1} W_{\left\{B_{j}\right\}}^{2 m, p}(\Omega): A \phi \in C^{\mu}(c l \Omega)\right\}
$$

is contained in $C^{2 m+\mu}(c l \Omega)$ and:

$$
\|\phi\|_{C^{2 m+\mu}(c l \Omega)} \leq \text { const }^{\prime}\left(\|A \phi\|_{C^{\mu}(c l \Omega)}+\|\phi\|_{C^{0}(c l \Omega)}\right), \quad \phi \in \mathscr{D} .
$$

These basic tools will be very useful for the study of regular solutions of (1).

\section{Regularity result}

\subsection{Main Theorems.}

To simplify further notation take that $Y$ denotes a space (together with the topology) selected from the family of Banach spaces

$$
\mathscr{X}=\left\{X^{\mu} ; \mu \in\left(0, \mu_{0}\right)\right\} \cup\left\{D\left(A_{p_{0}}^{\alpha}\right) ; \alpha \in\left[\alpha_{1}, 1\right)\right\},
$$

where $X^{\mu}$ was introduced in (17). Additionally, when $Y \cap V$ appears in considerations as a metric space, then the topology in $Y \cap V$ will always be induced from $Y$.

Let all requirements of Assumptions $H_{1}-H_{4}$ be satisfied from now on. Then, for each $Y \in \mathscr{X}$ :

THEOREM 1. A $C^{0}$-semigroup $T(t)$ of solutions of the problem (1) is generated on $Y$. Moreover, $T(t): Y \rightarrow Y$ is completely continuous for $t>0$ and if, in addition, for some $V \subset Y$ a priori estimate (11) is asymptotically independent of $u_{0} \in V$ (i.e. condition (15) holds), then there is a bounded subset $\mathscr{P}_{1}$ of $Y$ attracting each point of $V$ with respect to $T(t): Y \rightarrow Y$, i.e.

$$
\text { dist }_{Y}\left(T(t) u_{0}, \mathscr{P}_{1}\right) \stackrel{t \rightarrow+\infty}{\longrightarrow} 0, u_{0} \in V .
$$

As a consequence of the above theorem and [7, Th. 3.4.8] we have immediately:

THeOREM 2 (Smoothing Property). If for some $Y^{\prime} \in \mathscr{X}$ a subset $V \subset Y^{\prime}$ exists such that

(a) a priori estimate (11) is asymptotically independent of $u_{0} \in V$,

(b) $V$ is closed in $Y^{\prime}$,

(c) $T(t) V \subset V, t \geq 0$, 
then the semigroup $T(t)$ restricted to the complete metric space $Y^{\prime} \cap V$ has a global attractor $\mathscr{A} \subset Y^{\prime} \cap V$ (in the topology of $Y^{\prime}$ ). Furthermore for each $Y \in \mathscr{X}$ such that $Y \subset Y^{\prime}, \mathscr{A}$ is also the global attractor for $T(t)$ restricted to a complete metric space $Y \cap V$ (in the topology of $Y$ ).

REMARK 3. As stated in Theorems 1, 2, under the usual assumptions on $f$ (cf. considerations of Rem. 2), (1) generates semigroup $T(t)$ on Hölder spaces whenever sufficient regularity ( 3 ) of the boundary $\partial \Omega$ and coefficients of operators $A, B_{j}$ is provided. Moreover, asymptotic independence of any weak $a$ priori estimate (11) of initial data $u_{0}$ is then a necessary and sufficient condition for the existence of a global attractor for $T(t)$ in a strong topology of Hölder space $C^{2 m+\mu}(c l \Omega)$.

In a special case when $V=Y=D\left(A_{p_{0}}^{\alpha}\right)$ for some $\alpha \in\left[\alpha_{1}, 1\right)$, the proof of Theorems 1, 2 reduces in the main part to direct verification of all the assumptions of Hale's theorem [7, Cor. 3.9.5, p. 55]:

Proposition 3. Suppose that $X_{1}, X_{2}$ are Banach spaces, and

(i) $X_{1}$ is compactly embedded in $X_{2}, X_{1}$ dense in $X_{2}$.

(ii) $T(t)=S(t)+U(t): X_{j} \rightarrow X_{j}, S(t): X_{j} \rightarrow X_{j}$ is a linear semigroup with norm $\leq K(t)$ and $K(t) \rightarrow 0$ as $t \rightarrow+\infty, j=1,2$.

(iii) $U(t): X_{2} \rightarrow X_{1}$ is continuous, and for any $\tau \geq 0$, if $B,\{U(t) B, 0 \leq t \leq \tau\}$ are bounded in $X_{2}$, then $\{U(t) B, 0 \leq t \leq \tau\}$ is bounded in $X_{1}$.

(iv) $T(t)$ is point dissipative in $X_{2}$.

Then there is a global attractor $\mathscr{A}$ for $T(t)$ in $X_{2}$ and $T(t)$ is bounded dissipative in $X_{1}$.

If, in addition,

(v) $U(t)$ is conditionally completely continuous in $X_{j}, j=1,2$, then $\mathscr{A} \subset X_{1}$ and is a global attractor for $T(t)$ in $X_{1}$.

Nevertheless, in applications $V$ may be a proper subset of $Y$, so that a global attractor for $T(t)$ can then be obtained only on a metric subspace $Y \cap V$ of $Y$ (this situation may be observed e.g. in the Cahn-Hilliard equation). For this purpose the proof of Theorems 1, 2 is slightly more complicated and, although the idea of [7, Sec. 3.9] is generally followed, in spite of the verification of assumptions required in Proposition 3 (Lemma 1-6 below) additional smoothing properties of the semigroup generated by (1) (Lemma 7-9) must also be considered. 
The following paragraph is devoted to the proof of Theorems 1,2 , which will be given in a sequence of lemmas.

2.2. Smoothing action of the semigroup $T(t)$.

As already mentioned in Section 1, the problem (1) can be studied in the abstract form (4) with parameters $p_{0}>n$ and $\alpha \in\left[\alpha_{1}, 1\right)$ satisfying (9), (14) (note that $\left.\alpha_{1} \geq \alpha_{0}\right)$. Also, a $D\left(A_{p_{0}}^{\alpha}\right)$-solution $u$ of (4) starting from $u_{0}$ is then given by a variation of constants formula (cf. [8, Chapt. 3]):

$$
u\left(t, u_{0}\right)=e^{-A_{p_{0}} t} u_{0}+\int_{0}^{t} e^{-A_{p_{0}}(t-s)} F_{p_{0}}\left(u\left(s, u_{0}\right)\right) d s
$$

We shall consider further three families of mappings $T(t), S(t), U(t)$, such that:

$$
T(t)=S(t)+U(t), \quad t \geq 0
$$

where

$$
\begin{gathered}
T(t) u_{0}=e^{-A_{p_{0}} t} u_{0}+\int_{0}^{t} e^{-A_{p_{0}}(t-s)} F_{p_{0}}\left(u\left(s, u_{0}\right)\right) d s \\
S(t) u_{0}=e^{-A_{p_{0}} t} u_{0}
\end{gathered}
$$

and

$$
U(t) u_{0}=\int_{0}^{t} e^{-A_{p_{0}}(t-s)} F_{p_{0}}\left(u\left(s, u_{0}\right)\right) d s
$$

According to Proposition 1, for each $\alpha \in\left[\alpha_{1}, 1\right), T(t): D\left(A_{p_{0}}^{\alpha}\right) \rightarrow D\left(A_{p_{0}}^{\alpha}\right), t \geq 0$, is a strongly continuous semigroup of $D\left(A_{p_{0}}^{\alpha}\right)$-solutions of (4). However, since $S(t)$ is an analytic semigroup on $L^{p_{0}}(\Omega)$, then $S(t): D\left(A_{p_{0}}^{\alpha}\right) \rightarrow D\left(A_{p_{0}}^{\alpha}\right), t \geq 0$, is also a strongly continuous semigroup of $D\left(A_{p_{0}}^{\alpha}\right)$-solutions for

$$
\left\{\begin{array}{l}
\dot{v}+A_{p_{0}} v=0, \quad t>0, \\
v(0)=u_{0}
\end{array}\right.
$$

and as a consequence, for each $u_{0} \in D\left(A_{p_{0}}^{\alpha}\right), U(\cdot) u_{0}$ as a difference $T(\cdot) u_{0}-S(\cdot) u_{0}$ must be a $D\left(A_{p_{0}}^{\alpha}\right)$-solution of

$$
\left\{\begin{array}{l}
\dot{w}+A_{p_{0}} w=F_{p_{0}}\left(T(t) u_{0}\right), \quad t>0 \\
w(0)=0
\end{array}\right.
$$


Furthermore, maps $T, S, U$ introduced above can be considered on the product $[0,+\infty) \times D\left(A_{p_{0}}^{\alpha}\right)$ and, as a result of $[8$, Th. 3.4.1],

$$
S, T, U:[0,+\infty) \times D\left(A_{p_{0}}^{\alpha}\right) \rightarrow D\left(A_{p_{0}}^{\alpha}\right) \text { are continuous. }
$$

It is also worth noting that (4), (32), (33) are abstract formulations of regular parabolic initial boundary value problems resulting from (1) and hence, to obtain a priori bounds, the linear theory of parabolic problems (cf. conditions (21)-(24)) is applicable whenever necessary compatibility conditions are satisfied and suitable boundedness of right sides and initial functions is provided.

LEMMA 1. $X^{\mu}$ is compactly embedded and dense in $D\left(A_{p_{0}}^{\alpha}\right)$ for each $\mu \in\left(0, \mu_{0}\right), \alpha \in\left[\alpha_{1}, 1\right)$.

Proof. Compactness of the embedding $X^{\mu} \subset D\left(A_{p_{0}}^{\alpha}\right)$ follows immediately from inclusions:

$$
X^{\mu} \underset{\text { cont. }}{\subset} C_{\left\{B_{j}\right\}}^{2 m+\mu}(c l \Omega) \underset{\text { cont. }}{\subset} D\left(A_{p_{0}}\right) \underset{\text { comp. }}{\subset} D\left(A_{p_{0}}^{\alpha}\right)
$$

(cf. [8, Th. 1.4.8.]). For the proof of density we recall that $D\left(A_{p}\right)=W_{\left\{B_{j}\right\}}^{2 m, p}(\Omega)$ and $A_{p}=A+k_{0} I$ where $k_{0}>0$ has been chosen independently of $p \in(0,+\infty)$ in such a way that $A+k_{0} I$ is an isomorphism from $D\left(A_{p}\right)$ onto $L^{p}(\Omega)$ (cf. [6, Th. 19.2, 19.3] or $\left[12\right.$, Th. 4.9.1]). Let us fix arbitrary $\psi_{0} \in W_{\left\{B_{j}\right\}}^{2 m, p_{0}}(\Omega)$, take $\phi_{0} \in L^{p_{0}}(\Omega)$ such that $\psi_{0}:=\left(A+k_{0} I\right)^{-1} \phi_{0}$ and consider any sequence $\left\{\phi_{n}\right\} \subset C_{0}^{\infty}(\Omega)$ convergent to $\phi_{0}$ in $L^{p_{0}}(\Omega)$. Since $\left\{\phi_{n}\right\}_{n=1}^{\infty} \subset \bigcap_{p>1} L^{p}(\Omega)$ then also $\psi_{n}:=\left(A+k_{0} I\right)^{-1} \phi_{n}$ belongs to $\bigcap_{p>1} W_{\left\{B_{j}\right\}}^{2 m, p}(\Omega)$ for each $n=1,2, \ldots$, and

$$
\psi_{n} \stackrel{D\left(A_{p_{0}}\right)}{\longrightarrow} \psi_{0}
$$

Furthermore, since $\left(A+k_{0} I\right) \psi_{n}=\phi_{n}$ is an element of $C_{0}^{\infty}(\Omega) \subset C^{\mu_{0}}(c l \Omega)$ for $n=1,2, \ldots$, then basing on the property (23) we obtain that

$$
\psi_{n} \in C_{\left\{B_{j}\right\}}^{2 m+\mu_{0}}(c l \Omega), \quad n=1,2, \ldots
$$

which proves density of $X^{\mu}$ in $D\left(A_{p_{0}}\right)$ (note also, that $\left(A+k_{0} I\right) \psi_{n}=0$ on $\partial \Omega$, which is important in case when $B_{0}$ has the order $m_{0}=0$; cf. Rem. 1). Since it is known that $D\left(A_{p_{0}}\right)$ is dense in $D\left(A_{p_{0}}^{\alpha}\right)$ (cf. [8, Th. 1.4.8]), the proof of Lemma 1 is completed.

LeMmA 2. For each $Y \in \mathscr{X}, S(t): Y \rightarrow Y$ is a linear $C^{0}$-semigroup and

$$
\|S(t)\|_{\mathscr{L}(Y)} \leq K(t)
$$


where $\mathscr{L}(Y)$ denotes a space of linear continuous mappings on $Y$ and $K(t) \rightarrow 0$ when $t \rightarrow+\infty$. Furthermore, if $\alpha \in\left[\alpha_{1}, 1\right)$ and $B$ is bounded in $D\left(A_{p_{0}}^{\alpha}\right)$, then

$$
S(\tau) B \text { is bounded in } C^{2 m+\mu}(\operatorname{cl} \Omega) \text { for each } \tau>0, \quad \mu \in\left(0, \mu_{0}\right) \text {. }
$$

Proof. Clearly if $Y$ is a fractional power space the assertion follows from analyticity of $S(t)$ and the condition (8) (cf. [8, Chapt. 1]). Considering Hölder's $Y$ and using to (32) the linear theory (21) we observe that

$$
S(t)\left(\bigcup_{\mu<v} C_{\left\{B_{j}\right\}}^{2 m+v}(c l \Omega)\right) \subset \bigcup_{\mu<v} C_{\left\{B_{j}\right\}}^{2 m+v}(c l \Omega), \quad \mu \in\left(0, \mu_{0}\right) .
$$

Also, for any $u_{0} \in X^{\mu}$ and a sequence $\left\{u_{n}\right\} \subset \bigcup_{\mu<\nu} C_{\left\{B_{j}\right\}}^{2 m+v}(c l \Omega)$ :

$$
\left\|S(\cdot) u_{n}-S(\cdot) u_{0}\right\|_{C^{1+(\mu / 2 m), 2 m+\mu}([0, T] \times c l \Omega)} \leq c(T)\left\|u_{n}-u_{0}\right\|_{C^{2 m+\mu}(c l \Omega)}, \quad T>0,
$$

so that if $u_{n} \rightarrow u_{0}$ in $C^{2 m+\mu}(c l \Omega)$ then according to (38):

$$
S(t) u_{n} \stackrel{C^{2 m+\mu}(c l \Omega)}{\longrightarrow} S(t) u_{0}, \quad t \geq 0
$$

Conditions (39), (37) thus imply that for each $\mu \in\left(0, \mu_{0}\right)$

$$
S(t)\left(X^{\mu}\right) \subset X^{\mu}, \quad t \geq 0,
$$

and hence, in order to verify that $S(t)$ is a linear $C^{0}$-semigroup when $Y$ is a Hölder space only continuity of $S(t)$ at zero needs to be shown.

Let us take any sequence $\left\{h_{n}\right\}_{n \in N} \subset(0, \tau]$ convergent to 0 . Using to the problem (32) linear estimate (21), for $u_{0} \in X^{\mu}$ and $v \in \bigcup_{\mu<\nu} C_{\left\{B_{j}\right\}}^{2 m+v}(c l \Omega)$ we have the inequality

$$
\left\|S\left(h_{n}\right) u_{0}-S\left(h_{n}\right) v\right\|_{C^{2 m+\mu}(c l \Omega)} \leq c(\tau)\left\|u_{0}-v\right\|_{C^{2 m+\mu}(c l \Omega)},
$$

and hence it suffices to prove continuity of $S(t)$ on the dense subset of $X^{\mu}$. When $v \in \bigcup_{\mu<\nu} C_{\left\{B_{j}\right\}}^{2 m+v}(c l \Omega)$ it is clear that $v \in C_{\left\{B_{j}\right\}}^{2 m+v}(c l \Omega)$ for some $v \in\left(\mu, \mu_{0}\right)$ and, applying again (21), we get the uniform with respect to $n \in N$ estimate:

$$
\left\|S\left(h_{n}\right) v\right\|_{C^{2 m+v}(c l \Omega)} \leq c(\tau)\|v\|_{C^{2 m+v}(c l \Omega)} .
$$

In the presence of a compact embedding $C^{2 m+v}(c l \Omega) \subset C^{2 m+\mu}(c l \Omega), v>\mu$, (41) guarantees that a sequence $\left\{S\left(h_{n}\right) v\right\}_{n \in N}$ is precompact in $X^{\mu}$. Since $S(t)$ is continuous on $D\left(A_{p_{0}}^{\alpha}\right)$, then

$$
S\left(h_{n}\right) v \stackrel{D\left(A_{p_{0}}^{\alpha}\right)}{\longrightarrow} v, \text { when } n \rightarrow \infty
$$


so that recalling (41), the sequence $\left\{S\left(h_{n}\right) v\right\}_{n \in N}$ (as precompact in $X^{\mu}$ and convergent to $v$ in $\left.D\left(A_{p_{0}}^{\alpha}\right)\right)$ must converge to $v$ in $X^{\mu}$, i.e. continuity of $S(t)$ at zero is justified.

To prove (35) for Hölder's $Y$ we fix any $\mu \in\left(0, \mu_{0}\right)$ and start from the Schauder's type estimate (24) used for $S(t) u_{0}$ with fixed $t>0$ :

$$
\left\|S(t) u_{0}\right\|_{C^{2 m+\mu}(c l \Omega)} \leq c_{2}\left(\left\|A_{p_{0}} S(t) u_{0}\right\|_{C^{\mu}(c l \Omega)}+\left\|S(t) u_{0}\right\|_{C^{0}(c l \Omega)}\right) .
$$

From the Sobolev Embedding $D\left(A_{p_{0}}^{1 / 2 m}\right) \subset C^{\mu}(c l \Omega)\left(\mu \in\left(0, \mu_{0}\right)\right)$ and the well known properties of analytic semigroups (cf. [8, Th. 1.4.3.]) we have further:

$$
\begin{aligned}
\left\|A_{p_{0}} S(t) u_{0}\right\|_{C^{\mu}(c l \Omega)} & \leq C\left\|A_{p_{0}} S(t) u_{0}\right\|_{D\left(A_{p_{0}}^{1 / 2 m}\right)} \\
& =\left\|A_{p_{0}}^{1 / 2 m} A_{p_{0}} S(t) u_{0}\right\|_{L^{p_{0}}(\Omega)} \leq C_{1+(1 / 2 m)} \frac{e^{-a t}}{t^{1+(1 / 2 m)}}\left\|u_{0}\right\|_{L^{p_{0}}(\Omega)},
\end{aligned}
$$

and analogously

$$
\left\|S(t) u_{0}\right\|_{C^{0}(c l \Omega)} \leq C\left\|A_{p_{0}}^{1 / 2 m} S(t) u_{0}\right\|_{L^{p_{0}}(\Omega)} \leq C_{1 / 2 m} \frac{e^{-a t}}{t^{1 / 2 m}}\left\|u_{0}\right\|_{L^{p_{0}}(\Omega)} .
$$

Inserting (44), (45) into (43) we verify immediately that $\|S(t)\|_{\mathscr{L}\left(X^{\mu}\right)} \rightarrow 0$ when $t \rightarrow+\infty$.

For the proof of (36) it suffices to note that since $S(t)$ is analytic on $L^{p_{0}}(\Omega)$ then $S(t) u_{0} \in D\left(A_{p_{0}}^{2}\right) \subset D\left(A_{p_{0}}^{1+(1 / 2 m)}\right)$ for each $u_{0} \in D\left(A_{p_{0}}^{\alpha}\right)$ (cf. [6, Th. 2.2]). Using the Sobolev Embedding (cf. [8, Th. 1.6.1]) we obtain:

$$
A_{p_{0}} S(t) u_{0} \in D\left(A_{p_{0}}^{1 / 2 m}\right) \subset C^{\mu}(c l \Omega), \quad t>0, \quad \mu \in\left(0, \mu_{0}\right),
$$

and therefore $A_{p_{0}} S(t) u_{0} \in \bigcap_{p>1} L^{p}(\Omega)$ for each $t>0$. However, the latter implies (note (7) and the choice of $k_{0}>0$ in (5)):

$$
S(t) u_{0}=\left(A+k_{0} I\right)^{-1}\left(A_{p_{0}} S(t) u_{0}\right) \in \bigcap_{p>1} W_{\left\{B_{j}\right\}}^{2 m, p}(\Omega)
$$

so that when $t>0$ and $\mu \in\left(0, \mu_{0}\right)$ are fixed, Schauder type estimate (24) can be used for $S(t) u_{0}$ with arbitrary $u_{0} \in D\left(A_{p_{0}}^{\alpha}\right)$ and inequality (43) follows. Condition (36) is then a consequence of the same estimates as in formulae (43)-(45). The proof of Lemma 2 is completed.

LEMMA 3. For each $\mu \in\left(0, \mu_{0}\right)$ there are $\alpha \in\left[\alpha_{1}, 1\right)$ and $\varepsilon>0$ such that when $u_{0} \in D\left(A_{p_{0}}^{\alpha}\right)$, then

$$
\left.F_{p_{0}}\left(T(\cdot) u_{0}\right) \in C^{\mu+\varepsilon, \mu+\varepsilon}([0, \tau]) \times c l \Omega\right), \quad \tau>0 .
$$


Moreover, for each $\tau>0$, arbitrary bounded subset $\mathscr{B}$ of $D\left(A_{p_{0}}^{\alpha}\right)$ and all $u_{0} \in \mathscr{B}$ :

$$
\left\|F_{p_{0}}\left(T(\cdot) u_{0}\right)\right\|_{C^{\mu+\varepsilon, \mu+\varepsilon}([0, \tau] \times c l \Omega)} \leq \operatorname{const}(\tau, \mu, \varepsilon, \alpha, \mathscr{B}) .
$$

Proof. For arbitrary $u_{0} \in D\left(A_{p_{0}}^{\alpha}\right)$ there exists a $D\left(A_{p_{0}}^{\alpha}\right)$-solution $u(t)=$ $T(t) u_{0}$ of (4), with bounded trajectory, i.e. (cf. [5, (8)]):

$$
\sup _{t \in[0, \infty)}\|u(t)\|_{D\left(A_{p_{0}}^{\alpha}\right)} \leq z_{1}\left(u_{0}\right)
$$

(note that $z_{1}$ in (47) is bounded on bounded subsets of $D\left(A_{p_{0}}^{\alpha}\right)$ ). From (47) and the Sobolev Embedding $D\left(A_{p_{0}}^{\alpha}\right) \subset C^{k+v}(c l \Omega), v \in\left(0,2 m \alpha-\left(n / p_{0}\right)-k\right)$ (see Assumption $H_{2}$ and note that $\left.\alpha \geq \alpha_{1} \geq \alpha_{0}\right)$ we have ${ }^{1}$

$$
\sup _{t \in[0, \infty)}\|u(t)\|_{C^{k+v_{\alpha}(c l \Omega)}} \leq z_{1}^{\prime}\left(u_{0}\right), \quad v_{\alpha} \in\left(0,2 m \alpha-\frac{n}{p_{0}}-k-\left[2 m \alpha-\frac{n}{p_{0}}-k\right]\right) .
$$

Next, thanks to (48) the nonlinear term $F_{p_{0}}$ (being the counterpart of the sum $\left.f+k_{0} I\right)$ becomes bounded on $(0,+\infty) \times \Omega$, so that when $\alpha$ is close to 1 the estimate (22) can be used to $D\left(A_{p_{0}}^{\alpha}\right)$-solution $u(t)=T(t) u_{0}$ of (4) with $q=p_{0}$ on each cylinder $(0, \tau) \times \Omega$ and we get

$$
\|u\|_{W_{p_{0}}^{1,2 m}((0, \tau) \times \Omega)} \leq C\left(\tau, u_{0}, p_{0}\right)
$$

which ensures that

$$
\|u\|_{C^{v_{0}, v_{0}}([0, \tau] \times c l \Omega)} \leq C\left(\tau, u_{0}, p_{0}\right), \tau>0
$$

where $v_{0}$ was defined in (2).

It follows next from properties of Hölder functions (cf. [9, Chapt. II, $\S 3$, Lem. 3.1]) that if for any $h:[0, \tau] \times c l \Omega \rightarrow R$ :

$$
\sup _{x \in c l \Omega}\|h(\cdot, x)\|_{C^{\delta_{1}}([0, \tau])} \leq M_{1}
$$

and

$$
\sup _{t \in[0, \tau]}\left\|\frac{\partial h}{\partial x_{i}}(t, \cdot)\right\|_{C^{\delta_{2}(c l \Omega)}} \leq M_{2}, \quad i=1, \ldots, n
$$

\footnotetext{
${ }^{1}$ For the purpose of specific examples it should be mentioned here that when $k<2 m-1$ then stronger than $(48), C^{2 m \alpha-\left(n / p_{0}\right)}(c l \Omega)$ estimate of the solution still holds.
} 
then also

$$
\left\|\frac{\partial h}{\partial x_{i}}\right\|_{C^{\left(\delta_{1} \delta_{2}\right) /\left(1+\delta_{2}\right),\left(\delta_{1} \delta_{2}\right) /\left(1+\delta_{2}\right)}([0, \tau] \times c l \Omega)} \leq \operatorname{const}\left(M_{1}, M_{2}, \delta_{1}, \delta_{2}\right), \quad i=1, \ldots, n .
$$

If $k>0$, then basing on (48), (50) and applying the above property $k$-times for $l=1, \ldots, k$, step by step, we verify that for each $\tau>0$ :

$$
D^{l} u \in C^{v_{v_{0} \alpha}, v_{v_{0} \alpha}}([0, \tau] \times c l \Omega), \quad l=0, \ldots, k, \quad v_{v_{0} \alpha}=\frac{v_{0} v_{\alpha}^{k}}{\left(1+v_{\alpha}\right)^{k}}
$$

Furthermore, since $\alpha \in\left[\alpha_{1}, 1\right)$ may be arbitrarily close to 1 then increasing $v_{\alpha}$ to $v_{0}$ in (54) we observe that Hölder exponents $v_{v_{0} \alpha}$ remain inside $\left(0, \mu_{0}\right)$ and become arbitrarily close to $\mu_{0}$ defined in (2). Hence, when $\mu \in\left(0, \mu_{0}\right)$ is fixed it is possible to find $\alpha(\mu) \in\left[\alpha_{1}, 1\right)$ for which (54) holds with $v_{v_{0} \alpha(\mu)}>\mu$, so that denoting $\varepsilon=v_{v_{0} \alpha(\mu)}-\mu$ condition (54) may be rewritten in the form:

$$
D^{l} u \in C^{\mu+\varepsilon, \mu+\varepsilon}([0, \tau] \times c l \Omega), \quad l=0, \ldots, k, \quad \tau>0
$$

Next, (54) and Lipschitz continuity of $f$ lead to the condition

$$
f\left(\cdot, u, D^{1} u, \ldots, D^{k} u\right) \in C^{\mu+\varepsilon, \mu+\varepsilon}([0, \tau] \times c l \Omega), \quad \tau>0
$$

which gives immediately (note that $F_{p_{0}}$ is a substitution operator defined by the sum $f+k_{0} I$ whereas $u(t)$ was used to denote $\left.T(t) u_{0}\right)$ :

$$
F_{p_{0}}\left(T(\cdot) u_{0}\right) \in C^{\mu+\varepsilon, \mu+\varepsilon}([0, \tau] \times c l \Omega), \quad \tau>0
$$

Moreover, more precise calculations based on the Hölder property described in conditions (51)-(53) show that for each $\tau>0$ the estimate of a composite function $f\left(\cdot, u, D^{1} u, \ldots, D^{k} u\right)$ (and hence also of $\left.F_{p_{0}}\left(T(\cdot) u_{0}\right)\right)$ in the $C^{\mu+\varepsilon, \mu+\varepsilon}([0, \tau] \times c l \Omega$ ) norm is uniform with respect to $u_{0}$ varying in any bounded subset of $D\left(A_{p_{0}}^{\alpha(\mu)}\right)$, i.e. for each $\tau>0$ and arbitrary bounded subset $\mathscr{B}$ of $D\left(A_{p_{0}}^{\alpha(\mu)}\right)$ :

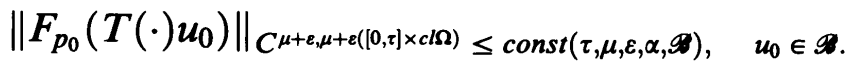

(This is in general a consequence of Assumption $H_{4}$ which implies that $z_{1}$ appearing in the condition (47) is bounded on bounded subsets of $D\left(A_{p_{0}}^{\alpha}\right)$ for $\alpha \in\left[\alpha_{1}, 1\right)$; cf. [5, Th. 1]). Lemma 3 is thus proved. 
LEMMA 4. For each $\mu \in\left(0, \mu_{0}\right)$ there is an $\alpha \in\left[\alpha_{1}, 1\right)$ such that $U:[0,+\infty) \times$ $D\left(A_{p_{0}}^{\alpha}\right) \rightarrow C_{\left\{B_{j}\right\}}^{2 m+\mu}(c l \Omega)$ is continuous. Moreover, for any $\tau>0$ and any bounded subset $B$ of $D\left(A_{p_{0}}^{\alpha}\right)$ the set $\bigcup_{0 \leq t \leq \tau}\{U(t) B\}$ is precompact in $C_{\left\{B_{j}\right\}}^{2 m+\mu}(c l \Omega)$.

Proof. For each $u_{0} \in D\left(A_{p_{0}}^{\alpha}\right)$ we have a $D\left(A_{p_{0}}^{\alpha}\right)$-solution $U(t) u_{0}=T(t) u_{0}-$ $S(t) u_{0}$ of the problem

$$
\left\{\begin{array}{l}
\dot{w}+A_{p_{0}} w=F_{p_{0}}\left(T(t) u_{0}\right), \quad t>0 \\
w(0)=0
\end{array}\right.
$$

Making use of Lemma 3, for fixed $\mu \in\left(0, \mu_{0}\right)$ we can choose $\alpha(\mu) \in\left[\alpha_{1}, 1\right)$ sufficiently close to 1 to ensure that for some $\varepsilon>0$,

$$
F_{p_{0}}\left(T(\cdot) u_{0}\right) \in C^{\mu+\varepsilon, \mu+\varepsilon}([0, \tau] \times c l \Omega), \quad \tau>0, \quad u_{0} \in D\left(A_{p_{0}}^{\alpha(\mu)}\right) .
$$

Since the problem (59) starts from zero then linear estimate (21) used to (59) in the presence of (60) guarantees that for each $\tau>0, u_{0} \in D\left(A_{p_{0}}^{\alpha(\mu)}\right)$ :

$$
U(\cdot) u_{0} \text { is a } C^{1+((\mu+\varepsilon) / 2 m), 2 m+\mu+\varepsilon}([0, \tau] \times c l \Omega) \text { solution of (59), }
$$

(note restrictions (19), (20) imposed on $A$ and $f$ in Rem. 1 in case when $B_{0}$ has the order $\left.m_{0}=0\right)$. Furthermore, according to Lemma 3, for each $\tau>0$ and any bounded subset $\mathscr{B}$ of $D\left(A_{p_{0}}^{\alpha(\mu)}\right)$ :

$$
\left\|U(\cdot) u_{0}\right\|_{C^{1+((\mu+\varepsilon) / 2 m), 2 m+\mu+\varepsilon}([0, \tau] \times c l \Omega)} \leq \operatorname{const}_{1}(\tau, \mu, \varepsilon, \alpha, \mathscr{B}), \quad u_{0} \in \mathscr{B} .
$$

From (62),

$$
\bigcup_{0 \leq t \leq \tau}\{U(t) B\} \text { is bounded in } C^{2 m+\mu+\varepsilon}(c l \Omega)
$$

and hence, compactness of $U$ follows immediately from compactness of the embedding $C^{2 m+\mu+\varepsilon}(c l \Omega) \subset C^{2 m+\mu}(c l \Omega)$.

In order to prove continuity of $U:[0,+\infty) \times D\left(A_{p_{0}}^{\alpha}\right) \rightarrow C_{\left\{B_{j}\right\}}^{2 m+\mu}(c l \Omega)$ it suffices to note that because of $(34)$

$$
U:[0,+\infty) \times D\left(A_{p_{0}}^{\alpha(\mu)}\right) \rightarrow D\left(A_{p_{0}}^{\alpha(\mu)}\right) \text { is continuous }
$$

and, as a consequence of the first part of this proof, for each $\tau>0$ and any bounded subset $\mathscr{B}$ of $D\left(A_{p_{0}}^{\alpha(\mu)}\right)$

$$
\bigcup_{0 \leq t \leq \tau}\{U(t) B\} \text { is precompact in } C^{2 m+\mu}(c l \Omega) \text {. }
$$


Since conditions (64), (65) ensure that $U\left(t_{n}, u_{n}\right) \rightarrow U\left(t_{0}, u_{0}\right)$ in $C^{2 m+\mu}(c l \Omega)$ whenever $\left(t_{n}, u_{n}\right) \rightarrow\left(t_{0}, u_{0}\right)$ in $[0,+\infty) \times D\left(A_{p_{0}}^{\alpha(\mu)}\right)$, the proof of Lemma 4 is completed.

Because $X^{\mu} \subset \bigcap_{\alpha \in(0,1)} D\left(A_{p_{0}}^{\alpha}\right)$, from the condition (61) of Lemma 4 it follows further that for each $\mu \in\left(0, \mu_{0}\right)$ there is $\alpha(\mu) \in\left[\alpha_{1}, 1\right)$ such that:

$$
\begin{aligned}
U(t)\left(X^{\mu}\right) & \subset U(t)\left(D\left(A_{p_{0}}^{\alpha(\mu)}\right)\right) \subset C_{\left\{B_{j}\right\}}^{2 m+\mu+\varepsilon}(c l \Omega) \\
& \subset c l_{C^{2 m+\mu}(c l \Omega)}\left(\bigcup_{\mu<\nu} C_{\left\{B_{j}\right\}}^{2 m+v}(c l \Omega)\right)=X^{\mu}, \quad t \geq 0 .
\end{aligned}
$$

Since also for all $\mu \in\left(0, \mu_{0}\right), \alpha \in\left[\alpha_{1}, 1\right)$ an embedding $X^{\mu} \subset D\left(A_{p_{0}}^{\alpha}\right)$ is compact and continuous, Lemma 4 implies immediately:

Lemma 5. For each $Y \in \mathscr{X}, U(t): Y \rightarrow Y$ is completely continuous for $t \geq 0$.

Recalling next that $T(t)=S(t)+U(t), t \geq 0$, we obtain from Lemmas 2, 4 required inclusions

$$
T(t)\left(X^{\mu}\right) \subset X^{\mu}, \quad t \geq 0, \quad \mu \in\left(0, \mu_{0}\right)
$$

and hence, since families $S(t): X^{\mu} \rightarrow X^{\mu}$ and $U(t): X^{\mu} \rightarrow X^{\mu}, t \geq 0$, have already been proved to be continuous, we obtain finally continuity of $T(t): X^{\mu} \rightarrow X^{\mu}$, $t \geq 0$, so that in the presence of (34) and Proposition 1:

Lemma 6. For each $Y \in \mathscr{X}, T(t): Y \rightarrow Y, t \geq 0$, is a $C^{0}$-semigroup of solutions of the problem (1).

Lemma 7. For each $Y \in \mathscr{X}, T(t): Y \rightarrow Y$, is completely continuous for $t>0$. Moreover, for each $\mu \in\left(0, \mu_{0}\right)$ there is $\alpha(\mu) \in\left[\alpha_{1}, 1\right)$ such that whenever $\alpha \in[\alpha(\mu), 1)$ and $B$ is a bounded subset of $D\left(A_{p_{0}}^{\alpha}\right)$, then

$$
T(t) B \text { is precompact in } X^{\mu} \text { for } t>0 .
$$

Proof. In the presence of Proposition 2 only Hölder's $Y$ needs to be considered. Linear estimate (21) and Lemma 3 ensure that for each $\mu \in\left(0, \mu_{0}\right)$, $\tau>0$ and any bounded subset $B$ of $X^{\mu}, \bigcup_{0 \leq t \leq \tau} T(t) B$ is bounded in $X^{\mu}$. Next, 
from the condition (63) and Lemma 2, for each $\mu \in\left(0, \mu_{0}\right)$ there are $\alpha(\mu) \in\left[\alpha_{1}, 1\right)$ and $\varepsilon>0$ such that both $U(\tau) B$ and $S(\tau) B, \tau>0$ are bounded in $C^{2 m+\mu+\varepsilon}(c l \Omega)$ whenever $B$ is bounded in $D\left(A_{p_{0}}^{\alpha(\mu)}\right)$. Since $T(t)$ is the sum $S(t)+U(t)$ and the embedding $C^{2 m+\mu+\varepsilon}(c l \Omega) \subset C^{2 m+\mu}(c l \Omega)$ is compact, the proof of Lemma 7 is completed.

LeMMA 8. For each $\mu \in\left(0, \mu_{0}\right)$ and all $\alpha \in[\alpha(\mu), 1)$

$$
\omega_{D\left(A_{p_{0}}^{\alpha}\right)}\left(u_{0}\right)=\omega_{X^{\mu}}\left(u_{0}\right), \quad u_{0} \in D\left(A_{p_{0}}^{\alpha}\right)
$$

where $\omega_{Y}\left(u_{0}\right)$ denotes an $\omega$-limit set of $u_{0}$ with respect to the topology of $Y$.

Proof. Since the $X^{\mu}$ topology is stronger than that of $D\left(A_{p_{0}}^{\alpha}\right)$ (cf. Lemma $1)$, then $\omega_{X^{\mu}}\left(u_{0}\right) \subset \omega_{D\left(A_{p_{0}}^{\alpha}\right)}\left(u_{0}\right)$. Let us fix $\mu \in\left(0, \mu_{0}\right)$ and for $\alpha(\mu)$ as in Lemma 7 choose $\alpha \in(\alpha(\mu), 1)$ and take any $v_{0} \in \omega_{D\left(A_{p_{0}}^{\alpha}\right)}\left(u_{0}\right)$. The positive orbit $\gamma^{+}\left(u_{0}\right)$ is bounded in $D\left(A_{p_{0}}^{\alpha}\right)$ (cf. [5, Th. 1, (8)]), so that $T(1) \gamma^{+}\left(u_{0}\right)$ is precompact in $X^{\mu}$ as a result of Lemma 7. From the characterization of $\omega$-limit sets, $T\left(t_{j}\right) u_{0} \stackrel{D\left(A_{p_{0}}^{\alpha}\right)}{\longrightarrow} v_{0}$ for some sequence $\left\{t_{j}\right\}_{j \in N}, t_{j} \geq 1, t_{j} \rightarrow+\infty$. Since $\left\{T\left(t_{j}\right) u_{0}\right\}_{j \in N} \subset T(1) \gamma^{+}\left(u_{0}\right)$, then there is a subsequence $\left\{T\left(t_{j^{\prime}}\right) u_{0}\right\}$ convergent in $X^{\mu}$. The only possible limit of this is $v_{0}$, therefore $v_{0} \in \omega_{X^{\mu}}\left(u_{0}\right)$. Lemma 8 is proved.

LemMa 9. For each $Y \in \mathscr{X}$, if for some $V \subset Y$ an a priori estimate (11) is asymptotically independent of $u_{0} \in V$, then there is a bounded subset $\mathscr{P}_{1}$ of $Y$ attracting (with respect to $T(t): Y \rightarrow Y$ ) each point of $V$, i.e.

$$
\operatorname{dist}_{Y}\left(T(t) u_{0}, \mathscr{P}_{1}\right) \stackrel{t \rightarrow+\infty}{\longrightarrow} 0, \quad u_{0} \in V
$$

Proof. In the presence of Proposition 2 only Hölder's $Y$ needs to be considered. Thus, let $\mu \in\left(0, \mu_{0}\right)$ be fixed and $Y=X^{\mu}$. According to Lemma 1 we then know that $V$, as a subset of $X^{\mu}$, must be contained in $D\left(A_{P_{0}}^{\alpha(\mu)}\right)$ with $\alpha(\mu)$ as in Lemma 7. From Proposition 2 there is a bounded and closed subset $\mathscr{P}$ of $D\left(A_{p_{0}}^{\alpha(\mu)}\right)$ such that

$$
\operatorname{dist}_{D\left(A_{p_{0}}^{\alpha(\mu)}\right)}\left(T(t) u_{0}, \mathscr{P}\right) \rightarrow 0 \quad \text { when } \quad t \rightarrow+\infty
$$

Defining

$$
\mathscr{P}_{1}=\bigcup_{u_{0} \in V} \omega_{D\left(A_{p_{0}}^{\alpha(\mu)}\right)}\left(u_{0}\right)
$$


from [7, Th. 3.2.1] it is clear that $\mathscr{P}_{1}$ also attracts points of $V$ with respect to $T(t): D\left(A_{p_{0}}^{\alpha(\mu)}\right) \rightarrow D\left(A_{p_{0}}^{\alpha(\mu)}\right)$. Moreover, as a sum of invariant sets $\omega_{D\left(A_{p_{0}}^{\alpha(\mu)}\right)}\left(u_{0}\right), \mathscr{P}_{1}$ is itself invariant, i.e.

$$
T(t) \mathscr{P}_{1}=\mathscr{P}_{1}, \quad t \geq 0 .
$$

Hence, $\mathscr{P}_{1} \subset \mathscr{P}$ and therefore $\mathscr{P}_{1}$ is bounded in $D\left(A_{p_{0}}^{\alpha(\mu)}\right)$. In the presence of Lemmas $7,8, \mathscr{P}_{1}$ is thus precompact and, of course, bounded in $X^{\mu}$ and

$$
\mathscr{P}_{1}=\bigcup_{u_{0} \in V} \omega_{X^{\mu}}\left(u_{0}\right)
$$

Since for each $u_{0} \in V$ a positive orbit $\gamma^{+}\left(u_{0}\right)$ is bounded in $D\left(A_{p_{0}}^{\alpha(\mu)}\right)$ (cf. [5, Th. 1, (8)]), then by Lemma 7 ,

$$
T(1) \gamma^{+}\left(u_{0}\right)=\left\{T(t) u_{0}, t \geq 1\right\} \text { is precompact in } X^{\mu} .
$$

However, from the linear estimate (21), $\left\{T(t) u_{0}, t \in[0,1]\right\}$ is bounded in $X^{\mu}$, so that, recalling (68), the positive orbit $\gamma^{+}\left(u_{0}\right)$ is bounded in $X^{\mu}$. Referring in the presence of Lemma 7 to [7, Th. 3.2.1], we observe that $\bigcup_{u_{0} \in V} \omega_{X^{\mu}}\left(u_{0}\right)$ attracts each point of $V$ with respect to $T(t): X^{\mu} \rightarrow X^{\mu}$. The proof of Lemma 8 is finished.

Theorem 1 follows directly from Lemmas 6, 7, 9. For the proof of Theorem 2 note that if (26) holds with some nonempty $V$, then $V$ must contain a nonempty invariant and bounded subset of $Y^{\prime}$, so that from the condition (66) of Lemma 7 and Henry's estimate contained in the proof of [8, Th. 3.3.4], for each $Y \in \mathscr{X}$ the set $Y \cap V$ is nonempty. Moreover, $Y \cap V$ is closed in $Y$ whenever $Y \subset Y^{\prime}$. Thus for $Y \in \mathscr{X}, Y \subset Y^{\prime}$ the existence of a global attractor $\mathscr{A}_{Y \cap V}$ for $T(t)$ restricted to a metric space $Y \cap V$ (i.e. in a topology of $Y$ ) is a consequence of Theorem 1 and the general results of [7, Th. 3.4.8].

In order to prove that all attractors coincide, let us note first that since $Y \subset Y^{\prime}$ (cf. Lemma 1) then $\mathscr{A}_{Y \cap V}$ is bounded in $Y^{\prime}$ and therefore, since $\mathscr{A}_{Y \cap V}$ is invariant,

$$
\mathscr{A}_{Y \cap V} \subset \mathscr{A}_{Y^{\prime} \cap V} \quad \text { for } \quad Y \subset Y^{\prime}, \quad Y \in \mathscr{X} .
$$

It is also clear that $\mathscr{A}_{Y^{\prime} \cap V}$ must be bounded in $D\left(A_{p_{0}}^{\alpha_{1}}\right)$ (which is the largest space of the family $\mathscr{X}$ ), and hence an estimate similar to that in the proof of $[8, \mathrm{Th}$. 3.3.4] together with the condition (66) show that $\mathscr{A}_{Y^{\prime} \cap V}$ is bounded in $Y$ for any 
$Y \subset Y^{\prime}, Y \in \mathscr{X}$. However, the latter implies required inclusions

$$
\mathscr{A}_{Y^{\prime} \cap V} \subset \mathscr{A}_{Y \cap V} \text { for } Y \subset Y^{\prime}, \quad Y \in \mathscr{X},
$$

which in the presence of (69) finishes the proof of Theorem 2.

\section{Applications}

Comparing Theorems 1, 2 of the present paper with those of [5] it is easy to see that regularity results of Section 2 are applicable to all examples mentioned in [5, Sections 3, 4] with these limitations, that the present result has not been proved for systems but for a single equation and that for Dirichlet boundary conditions implication (20) should also hold. Hence, to avoid long citations, only a very brief description of the famous Cahn-Hilliard equation will be given here.

Cahn-Hilliard equation. Consider the problem (cf. [11, Chapt. III, $\S 4.2]$ ):

$$
(\mathrm{C}-\mathrm{H})\left\{\begin{array}{l}
w_{t}=\Delta\left[-\varepsilon^{2} \Delta w+\Phi^{\prime}(w)\right], \quad t>0, \quad x \in \Omega \\
\frac{\partial w}{\partial N}=\frac{\partial(\Delta w)}{\partial N}=0, \quad t>0, \quad x \in \partial \Omega \\
w(0, x)=w_{0}(x), \quad x \in \Omega,
\end{array}\right.
$$

where $\Omega$ is a bounded domain in $R^{n}(n \leq 3)$ having $C^{4+\text { Lipschitz }}$ regular boundary $\partial \Omega$ and

$$
\Phi(s)=a^{2} s^{2 b}+(\text { a polynomial of the order } \leq 2 b-1)
$$

with

$$
b \text { arbitrarily large if } n=1,2 \text { or } b=2 \text { if } n=3 \text {. }
$$

Since the triple $\left(\Delta^{2},\{\partial / \partial N, \partial(\Delta) / \partial N\}, \Omega\right)$ forms a regular elliptic boundary value problem cf. [6, Part 1, §19]), then for the exemplary choice of parameters $\alpha_{0}=(3 / 4)$ and $p_{0}=2 n$ requirements of Assumptions $H_{1}, H_{2}$ are satisfied with $\mu_{0}=1 / 18$. Next, the existence of the Lyapunov functional for $(\mathbf{C}-\mathbf{H})$ and the property that the spatial average $\bar{w}(t)=|\Omega|^{-1} \int_{\Omega} w(t) d x$ of any solution of $(\mathrm{C}-\mathrm{H})$ is independent of $t \geq 0$ allow us to obtain an a priori $H^{1}(\Omega)$ estimate of solutions of $(\mathrm{C}-\mathrm{H})$ required in (11). As already shown in direct calculations of [4, Ex. 1], condition (70) gives admissible for validity of (12)-(13) growth of the nonlinear term $\Delta\left(\Phi^{\prime}(u)\right)$, so that Assumption $H_{3}$ holds with $l=1, r=2$. As a consequence, the estimate (14) is satisfied with any $\alpha_{1} \in(3 / 4,1)$ (cf. [4, Ex. 1] or more general 
results of [2, Cor. 4.2]) and fixing arbitrary $\varepsilon \in(0,1 / 4)$ we obtain that in this example:

$$
\mathscr{X}=\left\{X^{\mu} ; \mu \in\left(0, \frac{1}{18}\right)\right\} \cup\left\{D\left(A_{p_{0}}^{\alpha}\right) ; \alpha \in\left[\frac{3}{4}+\varepsilon, 1\right)\right\} .
$$

From Theorem 1 for each $Y \in \mathscr{X}$ a $C^{0}$-semigroup $T(t)$ of solutions of the problem (1) is generated on $Y$. However, since each constant function is a stationary solution of $(\mathrm{C}-\mathrm{H})$, the existence of a global attractor for $T(t)$ may be shown only on a proper metric subspace of $Y$. Defining for any $\xi>0$

$$
V_{\xi}:=\left\{w \in D\left(A_{2 n}^{(3 / 4)+\varepsilon}\right) ;|\bar{w}| \leq \xi\right\},
$$

it follows from considerations of [5, Ex. 1] that the introductory $H^{1}(\Omega)$ estimate of solutions of $(\mathrm{C}-\mathrm{H})$ is asymptotically independent of $u_{0} \in V_{\xi}$. Since also $T(t) V_{\xi} \subset V_{\xi}(t \geq 0)$ and $V_{\xi}$ is closed in $D\left(A_{2 n}^{(3 / 4)+\varepsilon}\right)$, then in the presence of Theorems 1,2 we obtain that for arbitrary $\xi>0$ :

CoRollary. For each $Y \in \mathscr{X}$ there is the global attractor $\mathscr{A}$ for the semigroup $T(t)$ generated by $(\mathrm{C}-\mathrm{H})$ on a metric space $Y \cap V_{\xi}$ (in the topology of $Y$ ). Moreover, $\mathscr{A}$ is independent of the choice of $Y \in \mathscr{X}$.

\section{Acknowledgment}

The authors are grateful to the Referee for a number of comments improving the redaction of the paper.

\section{References}

[1] R. A. Adams, Sobolev Spaces, Academic Press, New York, 1975.

[2] H. Amann, Global existence for semilinear parabolic systems, J. Reine Angew. Math. 360 (1985), 47-83.

[3] A. N. de Carvalho, J. G. Ruas-Filho, Global attractors for parabolic problems in fractional power spaces, SIAM J. Math. Analysis 26 (2) (1995), 415-427.

[4] J. W. Cholewa, T. Dlotko, Global solutions via partial information and the Cahn-Hilliard equation, in: Symposium on Singularities and Differential Equations, 1993, Banach Center Publications (PWN), Vol. 33, Warsaw, 1996, 39-50.

[5] J. W. Cholewa, T. Dlotko, Global attractor for sectorial evolutionary equation, J. Differential Equations, 125 (1996), 27-39.

[6] A. Friedman, Partial Differential Equations, Holt, Rinehart and Winston, New York, 1969.

[7] J. K. Hale, Asymptotic Behavior of Dissipative Systems, AMS, Providence, R. I., 1988.

[8] D. Henry, Geometric Theory of Semilinear Parabolic Equations, Springer-Verlag, Berlin, 1981.

[9] O. A. Ladyẑenskaja, V. A. Solonnikov, N. N. Ural'ceva, Linear and Quasilinear Equations of Parabolic Type, Nauka, Moscow 1967. 
[10] A. Lunardi, E. Sinestrari, W. von Wahl, A semigroup approach to the time dependent parabolic initial boundary value problem, Differential Integral Equations 6 (1992), 1275-1306.

[11] R. Temam, Infinite-Dimensional Dynamical Systems in Mechanics and Physics, Springer-Verlag, New York, 1988.

[12] H. Triebel, Interpolation Theory, Function Spaces, Differential Operators, Veb Deutscher Verlag, Berlin, 1978, also; North-Holland, Amsterdam, 1978.

(Tomasz Dlotko and Jan Cholewa)

Institute of Mathematics,

Silesian University, 40-007 Katowice, Poland

E-mail address, T. Dlotko:

tdlotko@gate.math.us.edu.pl 